\title{
Effects of long-term monotherapy with glimepiride vs glibenclamide on glycemic control and macrovascular events in Japanese Type 2 diabetic patients
}

\author{
Hirohisa Onuma ${ }^{1}$, Kouichi Inukai $^{{ }^{\star}}$, Masaki Watanabe ${ }^{2}$, Yoshikazu Sumitani $^{1}$, Toshio Hosaka $^{1}$, \\ Hitoshi Ishida ${ }^{1}$ \\ ${ }^{1}$ Third Department of Internal Medicine, Kyorin University School of Medicine, Tokyo, Japan; \\ *Corresponding Author: kinukai@ks.kyorin-u.ac.jp \\ ${ }^{2}$ Watanabe Medical Clinic, Hiroshima, Japan
}

Received 17 December 2013; revised 8 January 2014; accepted 15 January 2014

Copyright (C) 2014 Hirohisa Onuma et al. This is an open access article distributed under the Creative Commons Attribution License, which permits unrestricted use, distribution, and reproduction in any medium, provided the original work is properly cited. In accordance of the Creative Commons Attribution License all Copyrights (c) 2014 are reserved for SCIRP and the owner of the intellectual property Hirohisa Onuma et al. All Copyright (c) 2014 are guarded by law and by SCIRP as a guardian.

\section{ABSTRACT}

We investigated whether long-term glimepiride (GP) monotherapy improves insulin resistance and exerts a beneficial effect on beta cell function, as compared with glibenclamide (GC). One hundred Japanese Type 2 diabetic patients were randomly assigned to the GP $(n=50)$ or the GC $(n=50)$ group. During a 5-year monitoring period, patients received the indicated SU monotherapy, while changes in SU doses were allowed as needed to maintain HbA1C below $7.0 \%$. The GC group, in parallel with fasting insulin, showed a rapid homeostatic model assessment (HOMA)-R increase and maintained a high HOMA-R level. In contrast, HOMA-R in the GP group decreased continuously, from 2.9 at baseline to 1.8 at study completion. In the GC group, HOMA-b was markedly increased in the first 6 months, then gradually decreased through 18 months. While the HOMA- $\beta$ elevation in the GP group was more moderate than that in the GC group, HOMA- $\beta$ levels were maintained with a slight decrease. The cumulative macrovascular disease outcome was 1 for the GP and 7 for the GC group, showing a significant difference. These results suggest that glimepiride monotherapy markedly improved HOMA-R with moderate insulin stimulation, which may account for the difference in macrovascular disease development as compared with the group receiving glibenclamide.

\section{KEYWORDS}

Glibenclamide; Glimepiride; Macrovascular Events; HOMA-RI $\beta$

\section{INTRODUCTION}

Glimepiride (GP), a sulfonylurea (SU) derivative, has been widely used in the treatment of Type 2 diabetic patients in Europe and Asia. This agent has a mild effect on insulin secretion, with a hypoglycemic effect equivalent to that of glibenclamide (GC) [1]. Therefore, this SU is anticipated to have additional extrapancreatic effects, such that glimepiride has been designated as a $3^{\text {rd }}$ generation SU. Recent clinical studies also showed that glimepiride enhances insulin sensitivity, though it stimulates less insulin secretion than conventional $2^{\text {nd }}$ generation SUs [2-4]. As to these SUs, we previously reported that insulin resistance was significantly reduced by 6 months without improvements in glycemic control by switching from $2^{\text {nd }}$ generation SUs to glimepiride [4]. In vitro experiments also demonstrated glimepiride to enhance insulin sensitivity via activation of insulin mediated glycogen synthesis [5], enhanced glucose uptake [6] and activation of peroxisome proliferator-activated receptor (PPAR) g transcriptional activity [7-9].

Though glimepiride, as a $3^{\text {rd }}$ generation SU, appeared to have a very favorable profile and thus a strong claim to be the first treatment choice in patients requiring $\mathrm{SU}$ 
therapy in Asia and Europe, we have not yet obtained evidence as to tolerability regarding glycemic control or efficacy in preventing macrovascular disease a long term. Herein, we investigated, in Type 2 diabetic Japanese patients, whether long-term glimepiride treatment actually improves insulin resistance and maintains cell function, as compared with a $2^{\text {nd }}$ generation SU, glibenclamide. We also evaluated the incidence of macrovascular disease in both groups. In this study, we demonstrated that long-term glimepiride monotherapy improves homeostatic model assessment (HOMA)-R and exerts a decreased risk for macrovascular events, as compared with glibenclamide.

\section{MATERIALS AND METHODS}

\subsection{Study Design and Methods}

After obtaining approval from the Institutional Ethics Review Committees of the participating hospitals, a randomized, open-labeled multicenter clinical trial was performed in 100 Japanese patients with uncontrolled Type 2 diabetes. The subjects were assigned to receive glimepiride monotherapy (GP group: $\mathrm{n}=50$ ) or glibenclamide monotherapy (GC group: $n=50$ ). Patients whose latest HbA1C was more than $8.5 \%$ were recruited. None had previously been treated with anti-diabetic oral-hypoglycemic agents. None of the subjects had evidence of macrovascular diseases based on physical examination and electrocardiography. Patients with impaired hepatic function (serum AST/ALT > 40) or renal function (Serum Creatinine $>1.5$ ), with severe obesity (BMI $>30$ ) were excluded. During a 5-year monitoring period, patients received the indicated SU monotherapy, while changes in SU doses were allowed to a maximum dose (GP: $6 \mathrm{mg}$, GC: $7.5 \mathrm{mg}$ ) as needed to maintain HbA1c (NGSP) below 7.0\%. HbA1C, fasting plasma glucose (FPG), fasting insulin and body weight were measured every 6 months. Some patients, whose HbA1C level were more than $9.0 \%$ for 3 months or whose glycemic control was good and did not need SU treatment, were excluded from this study. FPG, HbA1c, immunoreactive insulin (IRI), total cholesterol, triglyceride, and HDLcholesterol were measured every 6 months using standard laboratory techniques. In addition, indexes considered to reflect insulin resistance (HOMA-R; FPG X IRI/405, HOMA-b; 360 X IRI/(FPG-63)) were calculated. Severe hypoglycemia is defined as an event with symptoms consistent with hypoglycemia and necessitating the assistance of another person. The primary endpoints were levels of FPG, HbA1c, HOMA-R and HOMA-b. The secondary endpoint was the occurrence of any of the following events; fatal and nonfatal myocardial infarction (MI), acute coronary syndrome (ACS) and stroke. All study subjects provided written informed consent.

\subsection{Statistical Analysis}

Data are presented as means \pm SEs. Log transformation of continuous variables was used when needed to satisfy distributional requirements for parametric tests. Differences in clinical characteristics were assessed using the unpaired-paired Student's $t$ test and a $\mathrm{p}$ value < 0.05 was considered statistically significant. As the number of subjects and outcomes were too small for analysis with a Cox proportional-hazard survival model or by the Kaplan-Meier method, the Extended Fisher's exact test was used to examine the significance of the association between two variables in a $2 \times 2$ contingency table. Statistical analyses were performed using Stat View software (Version 5.01; SAS Institute, Cary, NC).

\section{RESULTS}

Among 100 Japanese Type 2 diabetic patients, 23 patients (GP/GC group: 11/12) dropped out during the study period. As to the remaining 77 subjects, the baseline characteristics of those in each group are shown in Table 1. Before treatment, there were no significant differences in these parameters between the two groups. As eight patients dropped out due to the occurrence of macrovascular disease, 69 completed the 5-year study. Changes in FPG, HbA1c, fasting IRI, body mass index (BMI) and HOMA-R/b were documented in the remaining 69 patients (GP/GC group: 38/31). Mean monotherapy dosages were $1.8 \pm 0.1 \mathrm{mg}$ at 6 months and $2.6 \pm$ $0.2 \mathrm{mg}$ at study completion in the GP group, and $2.2 \pm$ $0.3 \mathrm{mg}$ at 6 months and $3.1 \pm 0.3 \mathrm{mg}$ at study completion in the GC group. No severe hypoglycemic events occurred in any group. Figure 1 shows the change of glycemic control values (Figure 1(a); mean $\triangle \mathrm{FPG}$, Figure 1(b); mean $\triangle \mathrm{HbA} 1 \mathrm{c}$ ) in each group during the 5 year study period. Mean FPG and HbA1c reached their lowest levels at 12 months after starting treatment, then gradually increased until completion of the study. Thus, throughout the study period, we observed significant lowering of HbA1c in the GP, as compared with the GC, group ( $\mathrm{p}=$ 0.006 ), while no significant difference was observed in the FPG levels of these groups at the end of the study ( $p$ $=0.06$ ). The difference in HbA1c levels between the two groups was presumably due to the high frequency of hypoglycemia with GC, which made strict glycemic control unattainable. Figure 2 shows the change of fasting IRI (Figure 2(a)) and BMI (Figure 2(b)) of each group during the 5 year study period. Both fasting IRI and BMI, which appear to reflect insulin resistance, showed marked differences in patterns between these two groups, i.e. fasting IRI and BMI of the GC group increased promptly and then gradually decreased, while those of the GP group were maintained at levels below those of the GC group. Thus, at the end of the study, no significant differences were observed in fasting IRI $(p=0.06)$ 
Table 1. Baseline characteristics of the subjects in each group.

\begin{tabular}{cccc}
\hline & $\begin{array}{c}\text { GC Group } \\
(\mathrm{n}=38)\end{array}$ & $\begin{array}{c}\text { GP Group } \\
(\mathrm{n}=39)\end{array}$ & p Value \\
\hline Male/Female & $24 / 14$ & $26 / 13$ & n.s. \\
Age (years) & $56.8 \pm 1.6$ & $57.3 \pm 1.4$ & n.s. \\
BMI (kg/m2) & $24.0 \pm 0.6$ & $23.8 \pm 0.5$ & n.s. \\
FPG (mg/dl) & $211.7 \pm 8.6$ & $206.5 \pm 12.9$ & n.s. \\
HbA1C (\%) (NGSP) & $9.70 \pm 0.27$ & $9.63 \pm 0.31$ & n.s. \\
Fasting IRI (IU/ml) & $6.6 \pm 1.0$ & $5.9 \pm 0.8$ & n.s. \\
Triglycerides (mg/dl) & $201.3 \pm 8.8$ & $186.6 \pm 8.4$ & n.s. \\
T-Chol (mg/dl) & $200.4 \pm 7.6$ & $194.0 \pm 8.0$ & n.s. \\
HDL-chol (mg/dl) & $40.3 \pm 2.3$ & $41.2 \pm 3.0$ & n.s. \\
\hline
\end{tabular}

Data are presented as means + SE. ${ }^{*}$ p value $<0.05$.

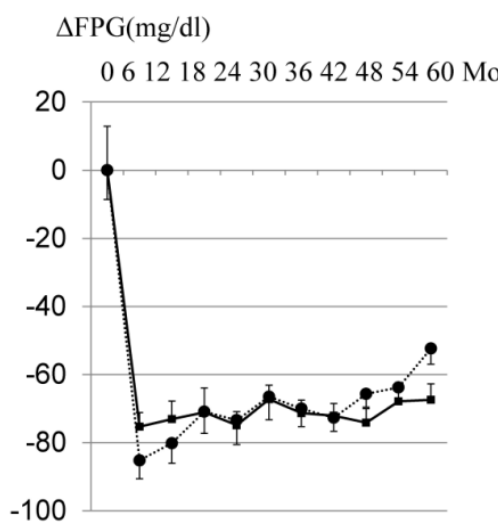

(a)

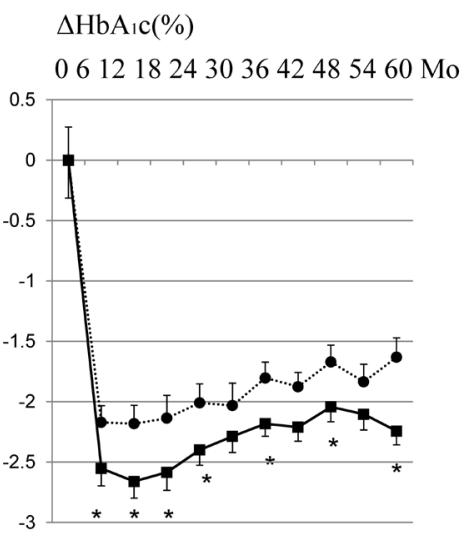

(b)

Figure 1. Time courses of FPG (a) and HbA1c (b) changes in the GP (closed squares) and GC (closed circles) groups during the 5-year treatment period. Data are presented as means \pm SE. ${ }^{*}$ p $<0.05$ compared with GC group.

and BMI between the two groups.

In the GC group, in parallel with fasting insulin, mean
$\Delta$ Fasting IRI (mU/l)

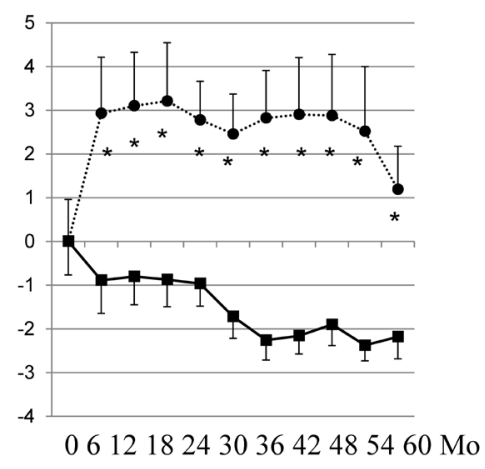

(a)

$\triangle \mathrm{BMI}\left(\mathrm{kg} / \mathrm{m}^{2}\right)$

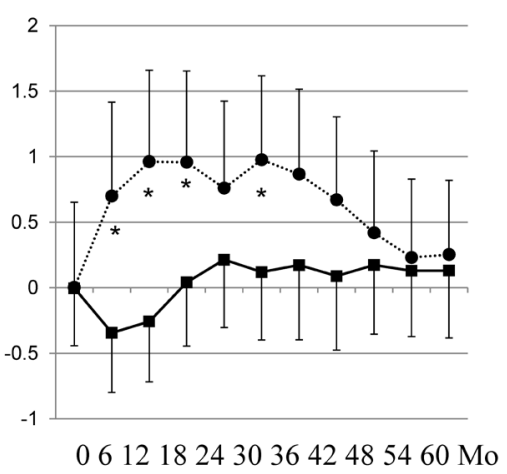

(b)

Figure 2. Time courses of fasting IRI (a) and BMI (b) changes in the GP (closed squares) and GC (closed circles) groups during the 5-year treatment period. Data are presented as means \pm SE. ${ }^{*} \mathrm{p}<0.05$ compared with GC group.

HOMA-R promptly increased, then gradually decreased after the peak at 18 months, until study completion, while in the GP group, HOMA-R decreased continuously (Figure 3(a)). These results clearly showed long-term GP treatment to actually improve insulin resistance. In the GC group, mean HOMA- $\beta$ increased markedly during the first 6 months, then gradually decreased (Figure 3(b)). On the other hand, HOMA- $\beta$ in the GP group exhibited a pattern different from that of the GC group. While the HOMA- $\beta$ elevation in the GP group was moderate, HOMA- $\beta$ levels were maintained with a slight decrease. These results suggest that the pleiotropic effects of GP, i.e. improved insulin resistance, lead to beta cell protection against insulin over-secretion. The macrovascular disease outcome was 1 for the GM and 7 for the GC group, showing a significant difference by Fisher's exact test (Table 2, $\mathrm{p}=0.023$ ). 


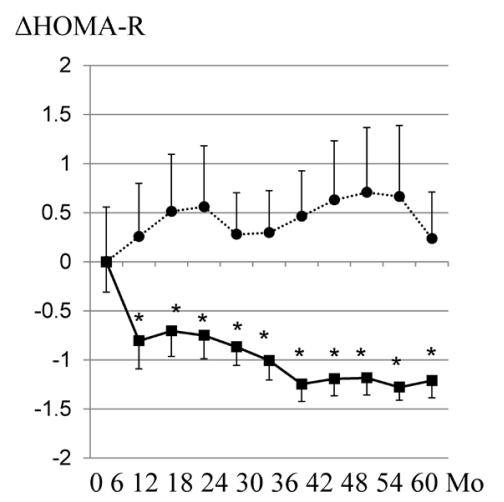

(a)

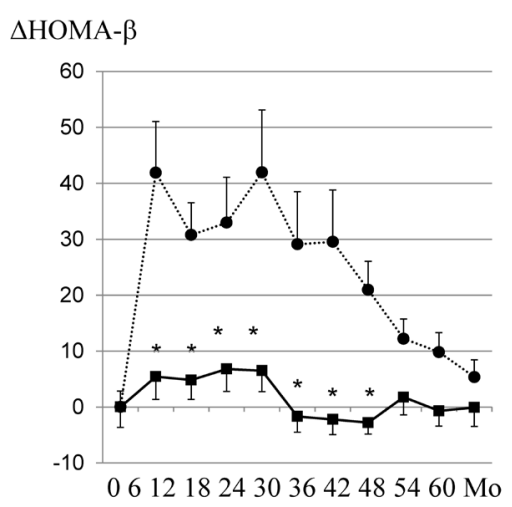

(b)

Figure 3. Time courses of HOMA-IR (a) and HOMA- $\beta$ (b) changes in the GP (closed squares) and GC (closed circles) groups during the 5-year treatment period. Data are presented as means $\pm \mathrm{SE}$. ${ }^{*} \mathrm{p}<0.05$ compared with GC group.

Table 2. The number of events in each group.

\begin{tabular}{ccc}
\hline & Events $(+)$ & Events $(-)$ \\
\hline GC Group & 7 & 31 \\
GP Group & 1 & 38 \\
\hline
\end{tabular}

Fisher's Exact Test: $\mathrm{p}=0.023$.

\section{DISCUSSION}

Glibenclamide has a high binding affinity for beta-cell SU receptors as compared with other SU agents [10], which is likely to be associated with the observed higher frequency of hypoglycemia [11]. Throughout the study period, we documented significant lowering of HbA1c in the GP, as compared with the GC group, which is probably due to the high frequency of hypoglycemia with GC making strict glycemic control unattainable. Despite the HbA1c difference, FPG levels of these two groups did not differ significantly. A possible explanation of this discrepancy might be that GP improves first-phase insulin secretion, which plays an important role in reducing postprandial glucose levels [12,13]. In fact, this addi- tional effect of GP may have contributed to the lowering of HbA1c levels in the GP group despite the FPG levels being similar to those of the GC group.

GP, a third generation SU, exhibits effects different from those of GC, including several extrapancreanic effects on muscle and adipose tissue, with elevation of active glucose transport and increased insulin secretion [14]. Our results also raise the possibility that GP enhances insulin sensitivity through activation of PPARg transcriptional activity [7]. Although the actual mechanism by which GP enhances insulin sensitivity has not been determined, recent clinical studies clearly demonstrated that GP enhances insulin sensitivity, though it stimulates less insulin secretion than conventional $2^{\text {nd }}$ generation SUs [2-4]. The present results also showed GP to markedly reduce HOMA-IR (from 2.8 to 1.8) throughout the 5 year study period, which would be the first evidence obtained from such a long-term study. Though, at the end of this study, HOMA- $\beta$ levels did not differ significantly between the two groups, the curves of the time courses were completely different, i.e. in the GC group, $\beta$ cell function increased during the two initial years, then, decreased during the latter 3 years. These increases and decreases were similar to the findings of a previous study, which also investigated long-term (6 years) use of GC or chlorpropamide [15]. Considering that HOMA- $\beta$ in the GP group was sustained at moderate levels (30 - 37) without marked deterioration for 5 years, we can reasonably suggest that GP is superior to GC in sustaining $\beta$ cell function.

We found that GP significantly reduced the risk of macrovascular events in Type 2 diabetic subjects as compared with GC treatment, while this conclusion may be limited by study design, i.e. small numbers. We can explain this difference as follows: as hyperglycemia is a significant predictor of macrovascular events [16], more favorable glycemic control, especially postprandial glucose lowering effects, in the GP group, had a beneficial effect on macrovascular events. Second, insulin signaling itself followed by Akt activation was recently demonstrated to be detrimental to the survival of human endothelial cells [17]. This leads to the other reason for the difference in HOMA-R, i.e. hyperinsulinemia raises susceptibility to macrovascular diseases. Finally, a previous report [18] clearly showed, by in vitro experimentation, that only GC blocks mitochondrial ATP-sensitive K+ channels in cardiac myocytes, resulting in the inhibition of ischemic preconditioning. In contrast, other SUs, GP or gliclazide, had no effects on the function of mitochondrial ATP-sensitive $\mathrm{K}+$ channels. Further study is anticipated to clarify the mechanism underlying the risk difference between the two SU agents in this long-term follow-up study. 


\section{CONCLUSION}

In conclusion, long-term GP monotherapy markedly improved HOMA-R with moderate insulin stimulation, thereby maintaining good glycemic control (HbA1c) in SU naïve diabetic patients, so does GC monotherapy. In addition, we observed a significant difference in macrovascular disease incidences between these two SUs when administered alone, suggesting that GP is superior to the conventional SU in terms of reducing the risk of macrovascular diseases.

\section{REFERENCES}

[1] Rosak, C. (2002) The pathophysiologic basis of efficacy and clinical experience with the new oral antidiabetic agents. Journal of Diabetes and its Complications, 16, 123-132. http://dx.doi.org/10.1016/S1056-8727(01)00207-0

[2] Rosenstock, J., Schneider, J., Samols, E. and Mushmore, D.B. (1996) Glimepiride, a new once-daily sulfonylurea. Diabetes Care, 19, 1194-1199. http://dx.doi.org/10.2337/diacare.19.11.1194

[3] Rosskamp, R., Wernicke-Panten, K. and Draeger, E. (1996) Clinical profile of the novel sulfonylurea glimepiride. Diabetes Research and Clinical Practice, 31, S33-S42. http://dx.doi.org/10.1016/0168-8227(96)01228-4

[4] Inukai, K., Watanabe, M., Nakashima, Y., Sawa, T., Takata, N., Tanaka, M., Kashiwabara, H., Yokota, K., Suzuki, M., Kurihara, S., Awata, T. and Katayama, S. (2005) Efficacy of glimepiride in Japanese Type 2 diabetic subjects. Diabetes Research and Clinical Practice, 68, 250257. http://dx.doi.org/10.1016/j.diabres.2004.10.002

[5] Haupt, A., Kausch, C., Dahl, D., Bachmann, O., Stumvoll, M., Haring, H.U. and Matthaei, S. (2002) Effect of glimepiride on insulin-stimulated glycogen synthesis in cultured human skeletal muscle cells; a comparison to glibenclamide. Diabetes Care, 25, 2129-2132. http://dx.doi.org/10.1016/j.diabres.2004.10.002

[6] Muller, G. and Wied, S. (1993) The sulfonylurea drug, glimepiride, stimulates glucose transport, glucose transporter translocation and dephosphorylation in insulin-resistant rat adipocytes in vitro. Diabetes, 42, 1852-1867. http://dx.doi.org/10.2337/diab.42.12.1852

[7] Inukai, K., Watanabe, M., Nakashima, Y., Takata, N., Isoyama, A., Sawa, T., Kurihara, S., Awata, T. and Katayama, S, (2005) Glimepiride Enhances Intrinsic Peroxisome Proliferator Activated Receptor-g Activity in 3T3L1 Adipocytes. Biochemical and Biophysical Research Communications, 328, 484-490. http://dx.doi.org/10.1016/j.bbrc.2004.12.190

[8] Fukuen, S., Iwaki, M., Yasui, A., Makishima, M., Matsuda, M. and Shimomura, I. (2005) Sulphonylurea agents exhibit peroxisome proliferator-activated receptor gamma agonistic activity. The Journal of Biological Chemistry, 280, 23653-23659.

http://dx.doi.org/10.1074/jbc.M412113200

[9] Marco, S., Podvinec, M., Roth, A., Hug, H., Kersten, S., Albrecht, H., Schwede, T., Meyer, U.A. and Rücker, C. (2007) Sulphonylureas and glinide exhibit peroxisome prolierator-activated receptor g activity: A combined virtual screening and biological assay approach. Molecular Pharmacology, 71, 398-406.

[10] Müller, G., Hartz, D., Pünter, J., Okonomopulos, R. and Kramer, W. (1994) Differential interaction of glimepiride and glibenclamide with the beta-cell sulfonylurea receptor I Binding characteristics. Biochimica et Biophysica Acta, 1192, 267-277. http://dx.doi.org/10.1016/0005-2736(94)90177-5

[11] Rendell, M. (2004) The role of sulfonylurea in the management of Type 2 diabetes mellitus. Drugs, 64, 13391358. http://dx.doi.org/10.2165/00003495-200464120-00006

[12] Feinböck, C., Luger, A., Klinger, A., Egger, T., Bielesz, G.K., Winkler, F., Siebenhofer, A., Grosschädl, F., Frank, E. and Irsigler, K. (2003) Prospective multicentre trial comparing the efficacy of and compliance with, glimepiride or acarbose treatment in patients with Type 2 diabetes not controlled with diet alone. Diabetes, nutrition \& metabolism, 16, 214-221.

[13] Korytkowski, M., Thomas, A., Reid, L., Tedesco, M.B., Gooding, W.E. and Gerich, L. (2002) Glimepiride improves both first and second phases of insulin secretion in Type 2 diabetes. Diabetes Care, 25, 1607-1611. http://dx.doi.org/10.2337/diacare.25.9.1607

[14] Muller, G.., Satoy, Y. and Geisen, K. (1995) Extrapancreatic effects of sulfonylurea-A comparison between glimepiride and conventional sulfonylurea. Diabetes Research and Clinical Practice, 28, S115-S137. http://dx.doi.org/10.1016/0168-8227(95)01089-V

[15] UK Prospective Diabetes Study Group (1995) Overview of 6 year's therapy of Type 2 diabetes: A progressive disease. Diabetes, 44, 1249-1258. http://dx.doi.org/10.2337/diab.44.11.1249

[16] Holman, R.R., Paul, S.K., Bethel, M.A., Matthews, D.R. and Neil, H.A. (2008) 10-year follow-up of intensive glucose control in Type 2 diabetes. The New England Journal of Medicine, 359, 1577-1589. http://dx.doi.org/10.1056/NEJMoa0806470

[17] Miyauchi, H., Minamino, T., Tateno, K., Kunieda, T., Toko, H. and Komuro, I. (2004) Akt negatively regulates the in vitro lifespan of human endothelial cells via a p53/ p21-dependent pathway. The EMBO Journal, 23, 212-220. http://dx.doi.org/10.1056/NEJMoa0806470

[18] Sato, T., Nishida, H., Miyazaki, M. and Nakaya, H. (2006) Effects of sulfonylureas on mitochondrial ATP-sensitive $\mathrm{K}^{+}$channels in cardiac myocytes: Implications for sulfonylurea controversy. Diabetes/Metabolism Research and Reviews, 22, 341-347. http://dx.doi.org/10.1002/dmrr.621 